\title{
Exploring Barriers to Medication Safety in an Ethiopian Hospital Emergency Department: A Human Factors Engineering Approach
}

\author{
Ephrem Abebe, PhD ${ }^{1}$; Michelle A. Chui, PharmD, PhD ${ }^{2}$ \\ ${ }^{1}$ The Johns Hopkins University; ${ }^{2}$ University of Wisconsin-Madison
}

\begin{abstract}
Objective: To describe challenges associated with the medication use process and potential medication safety hazards in an Ethiopian hospital emergency department using a human factors approach. Methods: We conducted a qualitative study employing observations and semi-structured interviews guided by the Systems Engineering Initiative for Patient Safety model of work system as an analytical framework. The study was conducted in the emergency department of a teaching hospital in Ethiopia. Study participants included resident doctors, nurses, and pharmacists. We performed content analysis of the qualitative data using accepted procedures.

Results: Organizational barriers included communication failures, limited supervision and support for junior staff contributing to role ambiguity and conflict. Compliance with documentation policy was minimal. Task related barriers included frequent interruptions and work-related stress resulting from job requirements to continuously prioritize the needs of large numbers of patients and family members. Person related barriers included limited training and work experience. Work-related fatigue due to long working hours interfered with staff's ability to document and review medication orders. Equipment breakdowns were common as were non-calibrated or poorly maintained medical devices contributing to erroneous readings. Key environment related barriers included overcrowding and frequent interruption of staff's work. Cluttering of the workspace compounded the problem by impeding efforts to locate medications, medical supplies or medical charts. Conclusions: Applying a systems based approach allows a context specific understanding of medication safety hazards in EDs from low-income countries. When developing interventions to improve medication and overall patient safety, health leaders should consider the interactions of the different factors.
\end{abstract}

Key words: Medication safety; Emergency departments; Low-income countries.

\section{INTRODUCTION}

Background

Emergency departments (EDs) are prone to experience frequent medication errors. ${ }^{1,2}$ Among the contributors include factors inherent to the practice of emergency medicine including: the fast paced nature of the work, unpredictability of patients' medical conditions and that of the clinical work flow as well as the frequent use of "high-risk" intravenous medications. ${ }^{2}$ Previous research has also shown that poorly designed technology ${ }^{3}$, physical workspace, and work processes in EDs can contribute to errors. ${ }^{4}$

While it is impossible to completely avoid medication errors, EDs in many high-income countries have successfully reduced medication error-related patient harm through a combination of interventions including, redesign of the physical workspace and work processes ${ }^{5}$, implementation of order review/medication reconciliation programs ${ }^{6}$, interdisciplinary collaboration and teamwork ${ }^{7}$, and use of health information technology (health IT)..$^{5,8,9}$ In contrast, many hospital EDs in low-income countries such as in Ethiopia are far from realizing the benefits of resource intensive interventions such as health IT. Nevertheless, there may be opportunities to improve medication safety in such settings through application of systems thinking and redesign of the patient care process.

Corresponding author: Ephrem Abebe, $\mathrm{PhD}$

The Johns Hopkins University

Email: ephremabebe@jhmi.edu

http://z.umn.edu/INNOVATIONS

2018, Vol. 9, No. 1, Article 7
Currently, the field of emergency medicine is slowly being introduced as a distinct specialization of medicine in Ethiopia. ${ }^{10}$ Although efforts to develop formal EDs in Ethiopian hospitals began in the early 2000s, a major impetus for such development came from the Ethiopian Hospital Management Initiative-an initiative launched through a partnership between the Ethiopian Federal Ministry of Health (FMOH), Clinton Health Access Initiative (CHAI) and Global Health Leadership Institute at Yale University. ${ }^{11}$ One key output of this initiative was the Ethiopian Hospital Reform Implementation Guideline. Introduced in 2010, this guideline served as a blueprint- and consolidated support for reorganizing hospital services into three major areas: in-patient, ambulatory, and emergency services.

Formalizing the delivery of emergency medical services, many hospitals in the country have since established EDs, though their capacity in terms of human resource and infrastructure remains far from optimal. During emergencies, patients are transported to such EDs by family members and friends using taxis (about $60 \%$ ) or personal vehicles, or by ambulances (about $14 \%$ ). ${ }^{12}$ After presenting to EDs, patients are triaged primarily by nurses who will then hand them over to physicians. After initial evaluation, patients may be discharged, transferred to other institutions, admitted to in patient wards, or stay in the ED for a short observation period.

In Ethiopia, trauma caused by traffic accidents is a major cause of patient visit to EDs. Because of the weak prehospital care 
services, such patients often arrive in EDs long after their injury. One study showed that only $19 \%$ of patients arrived at the ED of a major referral hospital within one hour of their injury. ${ }^{12}$ Other common causes for ED visits include diarrheal illnesses, medical emergencies, malaria, and complications of HIV/AIDS such as tuberculosis. Often, hospital EDs are also the first point of contact for patients with non-urgent medical conditions, adding further strain to the meager ED resources in the country.

\section{Importance}

Evidence from healthcare settings of developed countries suggests that human-factors engineering approaches can effectively be used to identify and mitigate risks that may result from medication errors occurring in EDs. ${ }^{13}$ It is not known if such approaches can be applied to identify medication safety risks in EDs of low-income countries. Currently, healthcare leaders in Ethiopia are primarily focused on building capacity through development of the emergency medicine workforce and the physical infrastructure that will be used to provide emergency medical care ${ }^{14}$ However, it is also important to understand patient care experiences and barriers that impact patient outcomes, which are partly impacted by medication errors. Since ED environments are highly vulnerable to medication errors, a deeper examination of potential barriers to medication safety may provide an insight that can be used to develop interventions that will lead to improvements in medication safety in EDs of such environments.

\section{Goals of this Investigation}

Our goal in this research was to understand challenges associated with the medication use process and perceived barriers to medication safety in an Ethiopian ED using a systems approach. Towards this goal, we conducted observations and semi-structured interviews using a purposively sampled mix of healthcare professionals from an Ethiopian hospital ED.

\section{METHODS}

\section{Study Setting and Design}

This study was conducted in the ED of a tertiary-care teaching hospital in Ethiopia. The ED serves as a training site for students from the health professions including, nursing and medical students, interns, as well as residents from emergency medicine and other specialties.

The ED was formally established in 2009 with a capacity of 20 beds. It was constructed using prefabricated materials as an annex to the main hospital building, as there was no formal ED space in the hospital's original design. After being dropped off at the ED's front door, patients are carried into the ED and pass through a small triage area, which the healthcare workers (primarily nurses) use to assess acuity and severity of their conditions. Following triage, patients are generally handed off to resident doctors who will be waiting in the main treatment and stabilization area. Patients are then evaluated and given a presumptive diagnosis until a further workup can be performed. This area also contains patient beds each located against the walls and separated from each other by tarp curtains.

Following evaluation, and upon determination of a need for medications, the resident doctor writes an order for medications in the patient chart. While this seemed to be the commonest method of writing orders, such are also written using non-standard order forms including on the back of laboratory order slips, which may eventually sit loosely or be stapled with other sheets of paper within the patient's folder.

With the order written, medication dispensing primarily occurs at a small pharmacy space located within the ED. Dispensing by pharmacists is triggered whenever a patient's caregiver takes the patient folder and presents it to the pharmacist on shift. Upon locating the prescription order and verifying current date and the doctor's signature, the pharmacist notifies the caregiver of the medication(s) prices and instructs her to pay at a cashier's booth located just outside the ED. When presented with the receipt, the pharmacist dispenses the required medication(s) which the caregiver will hand to the nurse responsible administering medications. The ED also has an emergency crash cart located in one corner of the patient care area. If a patient does not have a caregiver, a nurse works with the pharmacists to obtain the required medications. If the required medication is out of stock, as was noted occasionally, caregivers will be required to purchase the medications from sources outside of the hospital-typically private pharmacies.

We employed a qualitative study design with direct, nonparticipant observations and semi-structured interviews using the Systems Engineering Initiative for Patient Safety (SEIPS) model of work system as a guiding framework for data collection and analysis (Figure 1). ${ }^{15}$ The SEIPS model is anchored in the disciplines of human factors and systems engineering-which examine performance of humans and their interactions with technology and the surrounding environment while acknowledging their limitations. ${ }^{16-19}$ According to the SEIPS model, a person (e.g., an ED nurse) performs different tasks (e.g., administration of medications) using various tools and technologies (e.g., medication administration sign-out sheet). These tasks occur within the immediate physical environment (e.g., the ED workspace) under some organizational constraints (e.g., policies guiding medication administration). The SEIPS model recognizes that medication errors in EDs occur not from solitary actions of individuals but rather due to systems that are conflicting, incomplete or sub-optimal which they are part of and with which they interact. ${ }^{15}$ A qualitative approach was followed in order to develop a rich understanding of the experiences of ED staff about potential barriers to medication safety. ${ }^{20}$ 


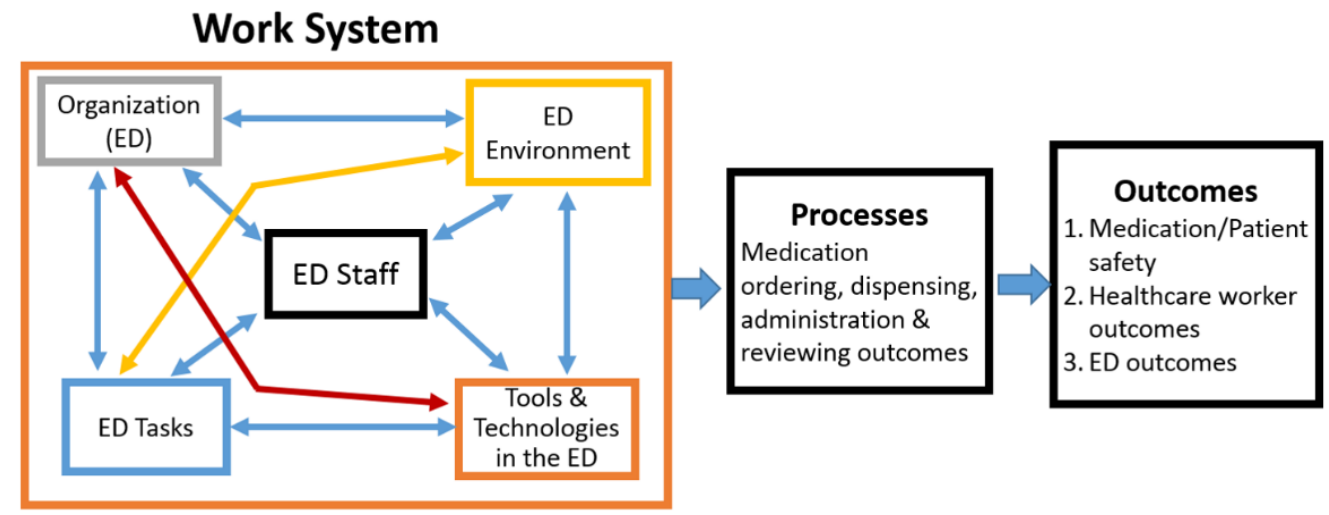

Figure 1: Adaptation of the Systems Engineering Initiative for Patient Safety Model of Work System to the Emergency Department (Adapted from Carayon et al. ${ }^{15}$ )

\section{Selection of Participants}

We used a purposive sampling to recruit ED staff that represent persons with different levels of work experience and professional background..$^{20} \mathrm{~A}$ total of 10 people -4 resident doctors, 4 nurses and 2 pharmacists-were approached and requested for participation by one of the researchers (EA). The attending physician in the ED provided guidance to recruit resident doctors working in the ED. Inclusion of resident doctors was considered essential as they were primarily responsible for most of the patient care and had the most intimate knowledge of the patient care process unlike the attending physician, who was not wholly available due to additional, non-clinical responsibilities. The charge nurse, who was responsible for coordinating the schedules of nursing staff, helped recruit nurses with varying levels of work experience in the ED. There were two pharmacists who were working in the ED pharmacy during the data collection period and both were included in the study. The study was approved by the Institutional Review Boards of the University of Wisconsin-Madison and that of the study site hospital. Informed consent was obtained from all participants.

\section{Data Collection}

We employed two methods of data collection using guides based on the SEIPS model ${ }^{15}$ addressing barriers to medication safety with respect to the five elements of the work system in the context of an Ethiopian ED. First, we observed participants at their job during which time one of the researchers (EA) took detailed, hand-written field notes. Each of the participants was observed for 4 to 6 hours at their job resulting in a total observation period from all participants of 40 hours. Next, we conducted in-depth semi-structured interviews with the same people whom we observed. Incidents and scenarios that were noted during the observations were used to elicit thoughts and facilitate discussions during the interviews. The interviews lasted 45 minutes to 1 hour and were conducted outside of the work hours of participants and in a quiet office location outside of the ED. All interviews were audio recorded and conducted in the local language, which were then translated into English and transcribed to facilitate analyses. Both the observation and interview guides were prepared and piloted by EA and MAC. EA received was trained in conducting observations and qualitative interviews and was responsible for all data collection. Further, EA is familiar with the culture and fluent in the local language. After data collection, each participant was given 200 Ethiopian Birr (the equivalent of $\$ 10$ US) as an incentive. All data were collected in August 2013.

\section{Data Analysis}

In preparation for the data analysis, observation notes and interview transcripts were read multiple times. The data were then imported into Nvivo $10^{\circledR}$ qualitative data analysis software (QSR International). Subsequently, the data were subjected to content analysis, ${ }^{21,22}$ by loosely following the five elements of the work system in the SEIPS model. EA performed a line by line coding of the data and generated an initial coding framework. These were then reviewed by both EA and MAC and an agreement was reached on the consistency of the codes generated. After all coding was completed, the codes were combined to form subcategories, which were then grouped to form the main themes later categorized under the each of the five elements of the work system in the SEIPS model. Significant phrases or statements that pertain to barriers to medication safety with respect to the five elements of the work system were identified. Credibility and study rigor was ensured through use of multiple sources of data and triangulating findings. 


\section{RESULTS}

\section{Characteristics of Study Participants}

Out of the 10 potential participants approached, 8 ( 2 resident doctors, 4 nurses, and 2 pharmacists) agreed to participate. Five participants were females while the rest were malestheir ages ranged between 22 and 35 years. Except for 1 participant, the majority had experience working in the ED for less than a year-from as little as 3 weeks to 12 months. To protect the identity of persons, we have deliberately avoided providing detailed demographic characteristics of our participants and information about their institution. In the next section, we describe the five major themes based on the work system model and provide illustrative verbatim quotes.

\section{Theme 1: Organization Related Barriers}

Organizational barriers that potentially impact medication safety in the ED were related to three key issuescommunication failures, hospital policy and procedures governing documentation of medication use in the ED, and lack of supervision and support to ED staff contributing to role conflict and ambiguity. The ED staff reported that problems with communication was a common problem and presented as a key barrier to medication safety for patients treated in the ED. This was reported to be an issue with both verbal and written communications. One of the resident doctors mentioned: [MD1] "We start patients suspected of having infections with board spectrum antibiotics, but once we narrow down the cause we will discontinue the antibiotic medication. But there are many instances where the patient will continue taking the medication even though it is discontinued already."

Verbal orders were particularly considered problematic and often resulted in duplication or omissions of treatment with medications. The second resident doctor mentioned: [MD2]: "Sometimes we tell the patient to stop a medication without necessarily writing it on the order sheet. So when the nurses come, they make the patient take the medication because they see it on the order sheet."
Furthermore, lack of clarity on issues and meanings related to order writing among resident doctors and nurses presented unique challenges, thus creating potentially unsafe conditions for patients receiving medications. [MD1]: "To be honest with you, there is even some confusion between residents and nurses on what the terms revised order, added order, or new order represent. We never officially discuss about these issues"

The ED currently uses a paper-based documentation system. Overall, there was little awareness and value for proper documentation of the patient care process in the ED and, more specifically, for documenting of medication orders. High workload was consistently mentioned as a primary reason for non-compliance with proper documentation. Poor documentation of medication orders resulted in increased workload on nurses who relied on the drug order sheets to carry out their medication-related tasks such as dispensing and administration of medicines. Nursing staff consistently stated that the practice of documenting drug orders in non-standard places in the patient folder was very common in the ED and it has made them engage in a continual habit of "hunting down" medication orders.

Our study also identified limited supervision and supportpartly due to critical shortage of senior staff-as potential barriers to medication and overall patient safety-which in turn led to role ambiguity and conflicts among the ED staff. Multiple opportunities develop for tasks to become antagonistic, especially among resident doctors. This was further compounded by the lack of coordination and communication as well as the differences in background training and unit culture that each of the resident doctor brought to the ED. Junior resident doctors mentioned lack of adequate support from their senior colleagues as one of the key obstacles interfering with proper patient care. At worst, most of the ED work was left to junior residents with minimal support from senior colleagues. Table 1 provides additional illustrative quotes from participants.

Table 1: Organization related barriers to medication safety

\begin{tabular}{|c|c|}
\hline \multirow{2}{*}{ Documentation Policy } & $\begin{array}{l}\text { [MD1] Resident doctor 1: "I have many patients waiting for me so I have to do this quickly. There is too much } \\
\text { paper in the patient folder and there isn't much time to find it [drug ordering sheet] and especially if it is a } \\
\text { returning patient, I won't be able to find the drug order sheet quickly because it [the drug order sheet] is hidden } \\
\text { somewhere in there. Therefore, I write the order at the back of any of the sheets or sometimes write in the plan } \\
\text { section of the medical chart. The nurses are used to this and will usually find it." }\end{array}$ \\
\hline & $\begin{array}{l}\text { [RN2] Nurse 2: "Here in the ED doctors often do not write their orders on the order sheet. Since we know that is } \\
\text { common, we first check the order sheet but don't stop there if there is nothing. It is common for them [the } \\
\text { residents] to write orders in the plan section, for example. So we go there too if there is nothing in the order sheet. } \\
\text { This practice is uncommon, though, in the inpatient wards but here it is common and most of us who work here } \\
\text { know it and do it. But for someone who is new here may be difficult until they get used to it." }\end{array}$ \\
\hline $\begin{array}{l}\text { Limited supervision and } \\
\text { support to junior staff }\end{array}$ & $\begin{array}{l}\text { [MD1] Resident doctor 1: "The senior residents mostly don't bother coming to the ED. They usually come in the } \\
\text { morning for the rounds and then leave. During a duty, it is usually the junior resident who stays in the ED } \\
\text { throughout the night while the senior stays in his residence apartment. They only come when there is a serious } \\
\text { condition and when we call them. Sometimes they don't even answer a call" }\end{array}$ \\
\hline
\end{tabular}




\section{Theme 2: Task Related Barriers}

Participants mentioned that tasks performed in the ED were often highly stressful in their nature (Table 2). Multiple factors played an additive role including - the need to juggle and continuously prioritize large numbers of patients needing attention, while addressing aggressive family members trying to advocate for their loved ones. The time sensitive nature of the care provided in the ED was also mentioned as a contributing factor to the stress and high task demands.

The work of ED staff also appeared to be frequently interrupted. Common causes included shortage of supplies, misplaced items-including medications and patient charts, as well as patients' family members and other hospital staff. We observed staff frequently breaking off from their current tasks-often multiple times within a span of few minutes-in order to locate and retrieve items needed in the care process. However, doing so was made difficult by the extremely cluttered workspace. Consequently, staff were sometimes forced to administer medications or write new orders without consulting the patient chart, and by only relying on reports from the patient or family members. On occasions, ED staff also failed to resume the interrupted tasks as they became tied to another emergent situation.

Table 2: Task Related Barriers to Medication Safety

\begin{tabular}{|c|c|}
\hline \multirow{2}{*}{$\begin{array}{l}\text { Stressful and time } \\
\text { sensitive tasks }\end{array}$} & $\begin{array}{l}\text { [RN1] Nurse 1: "This is really a not so good environment to work in. It's always a struggle dealing with the } \\
\text { patients and their family members. When, for example, I am working to administer a medication to a } \\
\text { patient, a caregiver of another nearby patient comes and asks me to do something because they may } \\
\text { perceive something is wrong. At times they turn violent and try to attack us. They can spit on your face. } \\
\text { We deal with all these issues and it's so stressful." }\end{array}$ \\
\hline & $\begin{array}{l}\text { [RN3] Nurse 3: "What we do here is mostly putting out the fire. It's difficult to work as a team because you } \\
\text { have to run around by yourself to attend to the needs of patients. We have lots of them [patients] here. } \\
\text { And the family members also don't understand our work here and they add to the stress. For example, } \\
\text { when I am seeing a patient, a caregiver for a patient on the next bed pokes me or says something in the } \\
\text { middle of my work [administering medications] and asks me to see his patient." }\end{array}$ \\
\hline $\begin{array}{l}\text { Frequent task } \\
\text { interruptions }\end{array}$ & $\begin{array}{l}\text { [MD1] Resident doctor 1: "As you noticed, we are overworked due to the high patient load. You are always } \\
\text { interrupted, called here and there-the doctor, the nurse, a family member. So it's really difficult to } \\
\text { maintain your composure and function well." }\end{array}$ \\
\hline
\end{tabular}

\section{Theme 3: Person Related Barriers}

All participants had limited experience working in an ED environment (Table 3). At the time of data collection, ED work experience among participant nurses ranged from 1 to 48 months. In addition, all pharmacy and nurse participants had no prior work experience or training to prepare them for the work in an ED environment. The ED was also staffed and primarily run by young resident doctors who had few weeks to 1 year of experience working in the ED. Nursing staff seemed to have the most difficulty especially during the beginning of their placement in the ED.

High staff turnover compounded the problem of limited experience. This was caused not only by staff leaving the hospital for other, better paying jobs, but also due to the hospital's policy of rotating staff-especially nurses-between different patient care units. All the nurse participants reported that they had worked mostly in inpatient wards and ambulatory clinics - environments completely different from an ED setting. Pharmacist participants did not seem to notice a major change in their work even though they recently started working in the satellite pharmacy located within the ED.

Fatigue resulting from long work hours was also commonly reported. Most reported working 10 or more hours was very common. The nurses reported that during a night duty, work hours usually last 14 hours which sometimes got prolonged due to a delayed handoff of patient care during a shift change. Resident doctors especially discussed the excessive workload as a common source of their fatigue interfering with their ability to communicate medication and other patient care related information to an incoming resident during a shift change. Although slightly variable, each resident doctor typically saw between 25 and 30 new patients during a half day period ( $8 \mathrm{am}-12 \mathrm{pm}$ ). These were in addition to the approximately 10 patients already in bed within the ED. Each nurse was also responsible for administering medications for the 10 patients. But they also assisted with new patients with different activities such as performing venipuncture, providing breathing support, as well as obtaining medications from the pharmacy and outside sources. 
Table 3: Person, Technology and Tools, and Work Environment Related Barriers to Medication Safety

\begin{tabular}{|c|l|}
\hline $\begin{array}{c}\text { Person Related Barriers } \\
\text { and experience }\end{array}$ & $\begin{array}{l}\text { [RN4] Nurse 4: "It is difficult at the beginning. Some of the drugs are new and I am not familiar with them. } \\
\text { I only learn about them from my seniors [senior staff nurses] who have been here for some time." }\end{array}$ \\
\hline $\begin{array}{c}\text { Work related } \\
\text { fatigue }\end{array}$ & $\begin{array}{l}\text { [MD2] Resident doctor 2: "It is usually the junior resident that does most of the work here [in the ED]. Also, } \\
\text { there is expectation from everyone like R2s and R3s [Year 2 and Year 3 residents] and seniors [attending } \\
\text { physicians] that we do the main work. Especially when on duty, we spend like 20 to 24 hours here [in the } \\
\text { ED]." }\end{array}$ \\
\hline $\begin{array}{c}\text { Poorly } \\
\text { maintained } \\
\text { technology/tools }\end{array}$ & $\begin{array}{l}\text { To help me fix the monitor. I remember once, triage monitor showed us a high BP [Blood Pressure] in a } \\
\text { patient who was hypovolemic despite the fact that she had signs of hypovolemia. Since we have the } \\
\text { experience of not trusting the equipment here we even do the manual measurement of the BP. So for that } \\
\text { lady, we eventually had to resuscitate her by giving her lots of fluids." }\end{array}$ \\
\hline \multicolumn{2}{|c|}{ Work Environment Related Barriers } \\
\hline $\begin{array}{c}\text { Crowding and } \\
\text { patient boarding }\end{array}$ & $\begin{array}{l}\text { [RN2] Nurse 2: "The Orthos [Orthopedics service] usually have a big problem with beds so their side has } \\
\text { many patients waiting here in the ED." }\end{array}$ \\
\hline
\end{tabular}

\section{Theme 4: Technology and Tools Related Barriers}

At the time of data collection, the ED had some basic equipment and technology including, oxygen cylinders, portable ultrasound and electrocardiogram machines, manual defibrillator, suction machines as well as blood pressure meter and pulse oximeters. Equipment breakdowns-mostly resulting from poor maintenance or use of outdated onesappeared to cause frequent task interruptions. Improper calibration or faulty readings from some of the equipment was also pointed out as a major concern (Table 3).

The nurse participants reported that they used a medication sign-out sheet as a useful tool to help them organize the medications to be administered during the administration rounds. In preparation for the medication administration rounds, nurses diligently transferred ordered medicationsalong with diagnoses-from each of the patient charts and kept them together in the sign-out sheet. During the observation periods, however, EA noted that the medication sign-out sheet contained medication-related information that was barely legible and-sometimes-conflicting. Most notably, the sign-out sheet was being re-used multiple times by erasing the entries from the previous 24-hour cycle. Our observation of this practice also revealed that incomplete erasures often left residual letters and numbers in the columns, potentially causing confusion when entries were made during the following 24-hour cycle.

\section{Theme 5: Work Environment Related Barriers}

Design of the ED's physical space presented key challenges with respect to having a conducive work environment for members of the ED. The interior was dimly lit as some of the fluorescent lamps were out of order and did not get replaced in time. This was particularly more challenging in the evening where there was no sufficient sunlight coming through the windows. We occasionally observed nurses using a flashlight from their personal cellphones to illuminate a patient's arm as they tried to perform a venipuncture.

Although located in the ED, pharmacists were physically separated from the rest of the ED staff and mostly communicated with caregivers and other members of the ED staff through a small window opening cut out of the pharmacy's door. Pharmacists worked behind the window to dispense medications when orders from the resident doctors were presented to them.

Participants described that the ED is mostly overcrowded due to high volume of patients and their accompanying caregivers. This finding appeared to be among the recurrent themes identified from the interviews and was confirmed by our observations. ED staff expressed their frustration that the overcrowding partly resulted from many patients using the ED for non-urgent conditions. Patient boarding was also mentioned as a problem adding to the crowding; due to unavailability of in-patient beds, many patients remained in the ED although a decision was made to admit them.

The ED workspace was extremely cluttered and this interfered with the work of staff in delivering safe care. Medical equipment (such as ultrasound and electrocardiogram machines, and portable oxygen cylinders) were all randomly placed across the ED work space-many of them contributing to safety hazards as healthcare professionals ran around in the ED caring for patients. Despite the best efforts to maintain order, patients' medical charts were often strewn across the space making it hard to locate them when needed. Most notably, the cluttered workspace also negatively impacted arrangement and storage of medications. For example, those kept in the emergency medicine crash cart were misplaced from their individually labeled drawer pockets, thus increasing the chances for their inadvertent administration during 
emergencies. Some of the medications that we noted to be misplaced or mislabeled during our observations include: $40 \%$ dextrose injection, calcium gluconate injection, potassium chloride injection, and thiopental sodium powder for injection-most with a high potential for fatal outcomes if given to the wrong patient or indication.

\section{DISCUSSION}

System improvement efforts that focus on improving medication and overall patient safety must begin with a thorough understanding of existing safety barriers. In this study, we used a systems engineering approach-the SEIPS model of work system ${ }^{15}$-to identify barriers influencing optimal medication use and safety within an Ethiopian ED setting. The overall impact of such barriers should be considered within the context of the whole ED and their interaction with each other. In doing so, researchers, hospital leaders, and policy makers can view the "whole picture" and develop a multi-pronged intervention to address the identified challenges instead of focusing on a single component, which, by way of priority setting, may diminish role of the other barriers.

Emergency departments are generally complex and dynamic with clinical work being highly uncontrolled and unpredictable. ${ }^{2}$ As a result, these environments tend to be more stressful. ${ }^{24}$ As was shown in our results, the drudgery involving a need to negotiate with the large number of patients and their caregivers, as well as the constant battle to navigate the work space and identify misplaced items-potentially worsened by clutter and the dimly lit work environment-can increase fatigue and make the work even more stressful for the ED staff. This, in turn, may contribute to increased job dissatisfaction for the ED staff-eventually forcing them to leave their work place, thus perpetuating the cycle of inexperience as new hires move in replacing those with better experiences.

Without diminishing the negative impact of severe resource deprivation, many of the identified challenges in the medication use process in our study are also likely to have their roots in the hospital (specifically, the ED) culture that may foster certain practices as accepted norms. And, as Aveling et al ${ }^{25}$ have noted, institutionally ingrained norms may be used to "legitimize or obscure poor practices that have the potential to cause patient harm." For example, the practice of multiple reuse of the medication administration chart to the extent it became so difficult to read medication names and their corresponding doses was treated as the normal part of the job. When asked to comment on the practice during the observation sessions, some of the nurses seemed to be indifferent and shrugged it off as "this is how we do it." Perhaps, this may also reflect apathy on the part of these professionals due to inadequate organizational support.
Many of the accounts from the study participants indeed highlighted significant levels of frustration due to poor organizational systems, which might explain the high turnover of staff (and hence the shortage) in the ED. And these mainly manifested in areas of professional development and staff retention as well as challenges related to rigid and hierarchical relationships between healthcare workers of the ED. In this regard, an important area of intervention for the hospital and ED leadership would be promoting a work culture that encourages respect among members of the ED staff as well as flattening unnecessary hierarchies to foster an enabling environment that allows open discussion of patient care concerns among the various members of the ED team.

Despite their good intentions, programs aimed at improving health system performance may have a very narrow focus and overlook critical processes that are essential to patient care and overall safety. ${ }^{26}$ The critical deficiencies identified in the medication delivery system of the ED illustrate this point. For example, while there was a great deal of emphasis on training staff in emergency care services (primarily physicians and nurses), work was limited in developing a medication use system that supports efficient delivery of medications. Our observation that family members were often responsible for obtaining the medications from the pharmacy shows the critical gap in this regard. A redesign of the current process that includes hospital personnel with ownership of the medication delivery could help reduce confusion and improve patient and family experiences while in the ED.

Our findings also showed that pharmacists' role in the ED was primarily limited to dispensing medications. Although having pharmacists formally assigned to work in the ED itself is a recent phenomenon in Ethiopian hospitals, their increased involvement in direct patient care presents an important opportunity to increase safety and quality of medication use. Even though the physical layout of the ED space limited the pharmacists' interactions with patients and other ED staff, redefining roles and designing teams to create a work environment where pharmacists actively interact with other ED members and contribute to medication reviews can be an important way to utilize the medication-related expertise of such professionals and improve overall patient safety. ${ }^{28}$

Developing a strong communication and teamwork can also contribute to building additional layers of safety into the medication use system and compensate for the inevitable weakness of healthcare professionals ${ }^{30}$-a fundamental tenet in the discipline of human factors engineering. ${ }^{16-19}$ For example, creating a standardized documentation system and a common language around medication use can be a good starting point for the ED and hospital leaders. This is particularly important because of the EDs place as a major training site for trainee resident doctors who may speak a "different language" than the nurses and pharmacists who are 
permanent employees of the hospital. Implementation of order sets-whenever appropriate-may further improve documentation by reducing some of the workload on ED staff, thus allowing improved compliance with hospital documentation policy. ${ }^{31}$

Clearly, an improvement effort that targets medication and patient safety in the ED must consider the realities of current context and the means available to ED and hospital leaders. To be successful, however, it is important to recognize the complexities surrounding medication use in the ED and the context under which medication use occurs - an intervention targeting one aspect of the medication use process will likely need to consider the direct and indirect influences of other contextual factors. For example, without decluttering the ED workspace and reducing the patient load, expecting ED staff to fully adhere to documentation requirements may result in little improvement.

Our study had some limitations that are worth mentioning. First, this was a single hospital study and thus generalizability of the findings may be limited. Nevertheless, like many qualitative studies, the goal of our study was not to make generalizations but rather to gain a deeper understanding of the personal experiences of ED staff with respect to perceived medication safety barriers. Second, the study had a small sample size and there is a potential that we may not have captured the full range of views from participants. For example, recruiting resident doctors was challenging as the institution was a training site and some of them had unpredictable work schedules. The more senior residents were mostly unavailable as most of the ED work was left for the junior residents, something that was commonly mentioned by the two resident doctors included in our study.

Third, most of our participants had at most 1 year of experience working in the ED. As a result, there may be a potential for missing some information regarding the work in the ED that would otherwise be gained by interviewing more experienced workers. In our experience, however, this did not seem to be an exception. At the time of data collection, the ED was being staffed mostly by young professionals with several months to few years of experience.

Finally, we were not able to include patient and family perspectives in this study. A future study should consider incorporating the views of this important group of stakeholders to develop even a more detailed understanding of medication safety barriers in the ED.

\section{CONCLUSIONS}

This study highlighted some of the challenges in the medication use process and potential barriers to medication safety in a low-income country's hospital ED. Applying a system-based approach allows a context specific understanding of medication safety hazards in EDs from lowincome countries. When developing interventions to improve medication and overall patient safety, health leaders should consider the interactions of the different factors. 


\section{References}

1. Jenkins G, Johnston PE, Patel NR, Jones I, Aronsky D. The epidemiology of medication prescribing errors in the emergency department. AMIA Annu Symp Proc. 2006:968.

2. Brown M. Medication Safety Issues in the Emergency Department. Crit Care Nurs Clin North Am. 2005;17(1):65-69. doi:10.1016/j.ccell.2004.09.009.

3. Ash JS, Berg M, Coiera E. Some unintended consequences of information technology in health care: the nature of patient care information systemrelated errors. J Am Med Inform Assoc. 2004;11(2):104-112. doi:10.1197/jamia.M1471.

4. Mitchell Scott B, Considine J, Botti M. Medication errors in ED: Do patient characteristics and the environment influence the nature and frequency of medication errors? Australas Emerg Nurs J. 2014;17(4):167-175. doi:10.1016/j.aenj.2014.07.004.

5. Aronsky D, Jones I, Lanaghan K, Slovis CM. Supporting Patient Care in the Emergency Department with a Computerized Whiteboard System. J Am Med Inform Assoc. 2008;15(2):184194. doi:10.1197/jamia.M2489.

6. Hayes BD, Donovan JL, Smith BS, Hartman CA. Pharmacist-conducted medication reconciliation in an emergency department. Am J Health Syst Pharm. 2007;64(16):1720-1723. doi:10.2146/ajhp060436.

7. Jones FD, Podila PM, Powers CD. Creating a Culture of Safety in the Emergency Department: The Value of Teamwork Training. J Nurs Adm. 2013;43(4):194200. doi:10.1097/NNA.0b013e31828958cd.

8. Rothschild JM, Churchill W, Erickson A, et al. Medication Errors Recovered by Emergency Department Pharmacists. Ann Emerg Med. 2010;55(6):513-521. doi:10.1016/j.annemergmed.2009.10.012.

9. Sard BE, Walsh KE, Doros G, Hannon M, Moschetti W, Bauchner H. Retrospective Evaluation of a Computerized Physician Order Entry Adaptation to Prevent Prescribing Errors in a Pediatric Emergency Department. Pediatrics. 2008;122(4):782-787. doi:10.1542/peds.2007-3064.

10. Bayleygne TM, Shahar A, Tsadic AW, et al. An international training program to assist with establishing emergency medicine in Ethiopia. Ann Emerg Med. 2000;36(4):378-382. doi:10.1067/mem.2000.110012.

11. Hartwig K, Pashman J, Cherlin E, et al. Hospital management in the context of health sector reform: a planning model in Ethiopia. Int J Health Plann Manage. 2008;23(3):203-218. doi:10.1002/hpm.915.
12. Meskere $Y$, Dinberu MT, Azazh A. Patterns and determinants of pre-hospital care among trauma patients treated in Tikur Anbessa Specialized Hospital, Emergency Department. Ethiop Med J. 2015;53(3):141-149.

13. Wears RL, Perry SJ. Human factors and ergonomics in the emergency department. Ann Emerg Med. 2002;40(2):206-212. doi:10.1067/mem.2002.124900.

14. Busse $\mathrm{H}$, Azazh A, Teklu S, et al. Creating change through collaboration: a twinning partnership to strengthen emergency medicine at Addis Ababa University/Tikur Anbessa Specialized Hospital--a model for international medical education partnerships. Acad Emerg Med. 2013;20(12):13101318. doi:10.1111/acem.12265.

15. Carayon P, Hundt AS, Karsh B-T, et al. Work system design for patient safety: the SEIPS model. Qual Saf Health Care. 2006;15(Suppl 1):i50-i58. doi:10.1136/qshc.2005.015842.

16. Karsh B-T, Holden RJ, Alper SJ, Or CKL. A human factors engineering paradigm for patient safety: designing to support the performance of the healthcare professional. Qual Saf Health Care. 2006;15(Suppl 1):i59-i65. doi:10.1136/qshc.2005.015974.

17. Carayon $P$, Karsh B-T, Gurses AP, et al. Macroergonomics in Healthcare Quality and Patient Safety. Rev Hum Factors Ergon. 2013;8(1):4-54. doi:10.1177/1557234X13492976.

18. Bromiley $M$. The journey of human factors in healthcare. J Perioper Pract. 2014;24(3):35-36.

19. Schaefer HG, Helmreich RL, Scheidegger D. Human factors and safety in emergency medicine.

Resuscitation. 1994;28(3):221-225. doi:10.1016/0300-9572(94)90067-1.

20. Creswell JW. Qualitative Inquiry and Research Design-Choosing Among Five Approaches. Third Edition. Los Angeles: SAGE Publications; 2013.

21. Elo $S$, Kyngäs $\mathrm{H}$. The qualitative content analysis process. J Adv Nurs. 2008;62(1):107-115. doi:10.1111/j.1365-2648.2007.04569.x.

22. Hsieh H-F, Shannon SE. Three Approaches to Qualitative Content Analysis. Qual Health Res. 2005;15(9):1277-1288. doi:10.1177/1049732305276687.

23. Abrahim O, Linnander E, Mohammed $\mathrm{H}$, Fetene $\mathrm{N}$, Bradley $E$. A patient-centered understanding of the referral system in Ethiopian primary health care units. PLoS One. 2015;10(10):e0139024. doi:10.1371/journal.pone.0139024. 
24. Källberg A-S, Göransson KE, Florin J, Östergren J, Brixey JJ, Ehrenberg A. Contributing factors to errors in Swedish emergency departments. Int Emerg Nurs. 2015;23(2):156-161. doi:10.1016/j.ienj.2014.10.002.

25. Aveling E-L, Parker M, Dixon-Woods $M$. What is the role of individual accountability in patient safety? A multi-site ethnographic study. Sociol Health IIIn. 2016;38(2):216-232. doi:10.1111/1467-9566.12370.

26. Assefa Y, Jerene D, Lulseged S, Ooms G, Damme WV. Rapid Scale-Up of Antiretroviral Treatment in Ethiopia: Successes and System-Wide Effects. PLOS Med. 2009;6(4):e1000056. doi:10.1371/journal.pmed.1000056.

27. Patanwala $A E$, Hays DP, Sanders AB, Erstad BL. Severity and probability of harm of medication errors intercepted by an emergency department pharmacist. Int J Pharm Pract. 2011;19(5):358-362. doi:10.1111/j.2042-7174.2011.00122.x.

28. Jacknin G, Nakamura T, Smally AJ, Ratzan RM. Using pharmacists to optimize patient outcomes and costs in the ED. Am J Emerg Med. 2014;32(6):673-677. doi:10.1016/j.ajem.2013.11.031.

29. Rollins $\mathrm{G}$. Medication errors in emergency department less likely to be intercepted before being administered. Rep Med Guidel Outcomes Res. 2003;14(8):1, 6-7.

30. Schmidt CE, Bottoni T. Improving medication safety and patient care in the emergency department. $J$ Emerg Nurs .2003;29(1):12-16.

31. Reingold S, Kulstad E. Impact of Human Factor Design on the Use of Order Sets in the Treatment of Congestive Heart Failure. Acad Emerg Med. 2007;14(11):1097-1105. doi:10.1111/j.15532712.2007.tb02395.x. 